\title{
Taste and odor in conditioned flavor preference learning
}

\author{
ELIZABETH D. CAPALDI and MARTIN J. HUNTER \\ University of Florida, Gainesville, Florida
}

\begin{abstract}
Almond and peppermint extracts were combined with salt and citric acid as cues in conditioned flavor preference conditioning. In Experiment 1, extracts overshadowed tastes, although tastes and extracts conditioned equally well when presented in isolation. In Experiments 2 and 3, tastes and extracts were conditioned in isolation prior to conditioning of a taste/extract compound. The conditioning history of the tastes and extracts did not affect the overshadowing of taste by extract. The results of Experiment 4 showed that rats could learn to discriminate between a taste and extract presented in isolation vs. the taste/extract compound. Thus, extracts do not interfere with sensing the tastes. We suggest that a taste/extract compound produces a configural stimulus that is more characteristic of the extract than the taste.
\end{abstract}

Animals can learn preferences for flavors associated with sweet tastes, calories, or other positive consequences (e.g., Capaldi, Campbell, Sheffer, \& Bradford, 1987; Holman, 1975). Despite many studies demonstrating conditioning of flavor preferences, there has been little work on the role of the components of flavor (i.e., taste and odor) in flavor preference conditioning. In contrast, there have been many studies of the role of taste and odor in aversion conditioning.

In aversion conditioning, taste is a strong cue when taste and odor are conditioned individually, whereas odor is a weak cue (e.g., Hankins, Garcia, \& Rusiniak, 1973). However, when odor and taste cues are combined, a strong aversion is formed to the odor (Rusiniak, Hankins, Garcia, \& Brett, 1979). This phenomenon has been termed potentiation. Taste potentiates aversion conditioning to odor in rats. Although not all investigators have observed potentiation (e.g., Bouton \& Whiting, 1982; Mikulka, Pitts, \& Philput, 1982), it has been found repeatedly by others (e.g., Durlach \& Rescorla, 1980; Palmerino, Rusiniak, \& Garcia, 1980; Rusiniak, Palmerino, Rice, Forthman, \& Garcia, 1982). In taste aversion, potentiation is asymmetrical; that is, odor does not potentiate conditioning to taste (Palmerino et al., 1980). This asymmetry has been related to the stronger conditioning obtained to taste than to odor when each is conditioned in isolation.

However, in flavor preference conditioning, strong conditioning is customarily obtained to odor conditioned in isolation (e.g., Holman, 1975). Perhaps, then, in conditioned preference learning, taste will not potentiate conditioning to odor. Alternatively, some theorists sug-

This research was supported in part by Grant MH 39453 to the first author from the National Institute of Mental Health. Requests for reprints should be sent to E. D. Capaldi, Department of Psychology, University of Florida, Gainesville, FL 32611-2065. gest that potentiation is a result of the special relationship between taste and odor in the feeding system (e.g., Garcia, 1989). Perhaps, then, taste will potentiate conditioning to odor in preference conditioning in a way that is similar to that in aversion conditioning. The purpose of Experiment 1 was to evaluate conditioned preferences for odor and taste when they had been conditioned in isolation or conditioned together.

\section{EXPERIMENT 1}

Food-deprived rats prefer a flavor paired with sucrose over a flavor paired with saccharin (e.g., Mehiel \& Bolles, 1984). We chose this preference for examination because it is robust.

In Experiment 1, we conditioned this preference by mixing sucrose and saccharin with tastes, extracts, or taste/extract compounds. Flavor extracts were given in water. Presumably, the extracts could be tasted as well as smelled. However, Rusiniak et al. (1979) found that rats react to extracts in water primarily on the basis of smell, not taste. Many researchers doing studies of potentiation in flavor-aversion learning have also used extracts in water as odor cues (Durlach \& Rescorla, 1980; Rusiniak et al., 1979; Rusiniak et al., 1982).

\footnotetext{
Method

Subjects. The subjects were 48 naive, male Sprague-Dawley rats from Harlan Co., Indianapolis, IN. They were 73 days old upon arrival and 83 days old at the start of deprivation.

Materials and Apparatus. Solutions (w/v) were presented in $50-\mathrm{ml}$ Nalgene centrifuge tubes with rubber stoppers and metal spouts. Taste stimuli were $.2 \% \mathrm{NaCl}$ and $.031 \%$ citric acid, purchased from Fisher Scientific, Fair Lawn, NJ. Odorous stimuli were McCormick's brand extracts: $1 \%$ peppermint extract or $1 \%$ almond extract. All stimuli were presented in $40 \mathrm{ml}$ of distilled water, which also contained either $3.42 \%$ sucrose or $.012 \%$ saccharin. The rats were trained and tested in their home cages. A 12:12-h light:dark cycle was always in effect, with lights on at 6:00 a.m. The subjects showed no reluctance to drink the solutions.
} 
Procedure. Water was present ad lib in clear plastic bottles mounted to the right of the food hopper on each cage front. On Day 1, all food was removed from the cages. On Days 2-11, the rats were fed $14 \mathrm{~g}$ each day at 9:00 a.m.; later, they were trained at this time each day. During training, the rats were fed at 11:30 a.m.

There were 10 days of training, beginning on Day 12. The 48 subjects were randomly divided into four groups of 12 subjects each. Group T was trained with the taste stimuli (salt and citric acid) - one taste mixed with sucrose, the other with saccharin. Group E was trained with the extracts (peppermint and almond) one extract mixed with sucrose, the other with saccharin. Groups TE-T and TE-E were both trained with one taste/extract combination mixed with sucrose, the other with saccharin. Groups $\mathrm{E}$ and TE-E were tested with the extracts; Groups $\mathrm{T}$ and TE-T were tested with the tastes.

In training, $40 \mathrm{ml}$ of solution were presented for $10 \mathrm{~min}$ each day. All stimuli were counterbalanced. For half of Group T, salt was mixed with sucrose and citric acid was mixed with saccharin; the other half had the reverse pairings. For half of Group E, almond was mixed with sucrose and peppermint with saccharin; the other half had the reverse pairings. For each TE group, 3 rats were given almond + citric acid in sucrose and peppermint + salt in saccharin, 3 received peppermint + citric acid in sucrose and almond + salt in saccharin, 3 received almond + citric acid in saccharin and peppermint + salt in sucrose, and the final 3 received peppermint + citric acid in saccharin and almond + salt in sucrose Sucrose and saccharin, with their associated cues, were given in an ABBA order over days.

Immediately following training, there were 2 days of testing. Groups $\mathrm{T}$ and TE-T were given a two-bottle test between salt and citric acid in water $(40 \mathrm{ml})$, and Groups $\mathrm{E}$ and TE-E were given a two-bottle test between almond and peppermint in water. Water bottles were removed during the test. For all the rats, the left tube was put into each cage and moved briefly so that the rat would approach and contact the tube. Then the right tube was inserted and moved briefly so that the rat would contact this tube as well. Each test lasted $4 \mathrm{~h}$. The positions of the tubes were reversed between tests. The subjects occasionally bit and shook the tubes during testing, but because they curved upward, these movements did not produce spillage. This behavior was not systematic, and it was directed toward the bottles containing cues that had been associated with either sucrose or saccharin.

\section{Results}

Training. The amount of each solution consumed by each group is shown in Figure 1. As can be seen in Figure 1, each group drank more of the sucrose solution than the saccharin solution, and this difference grew larger over days. An analysis of variance (ANOVA) included

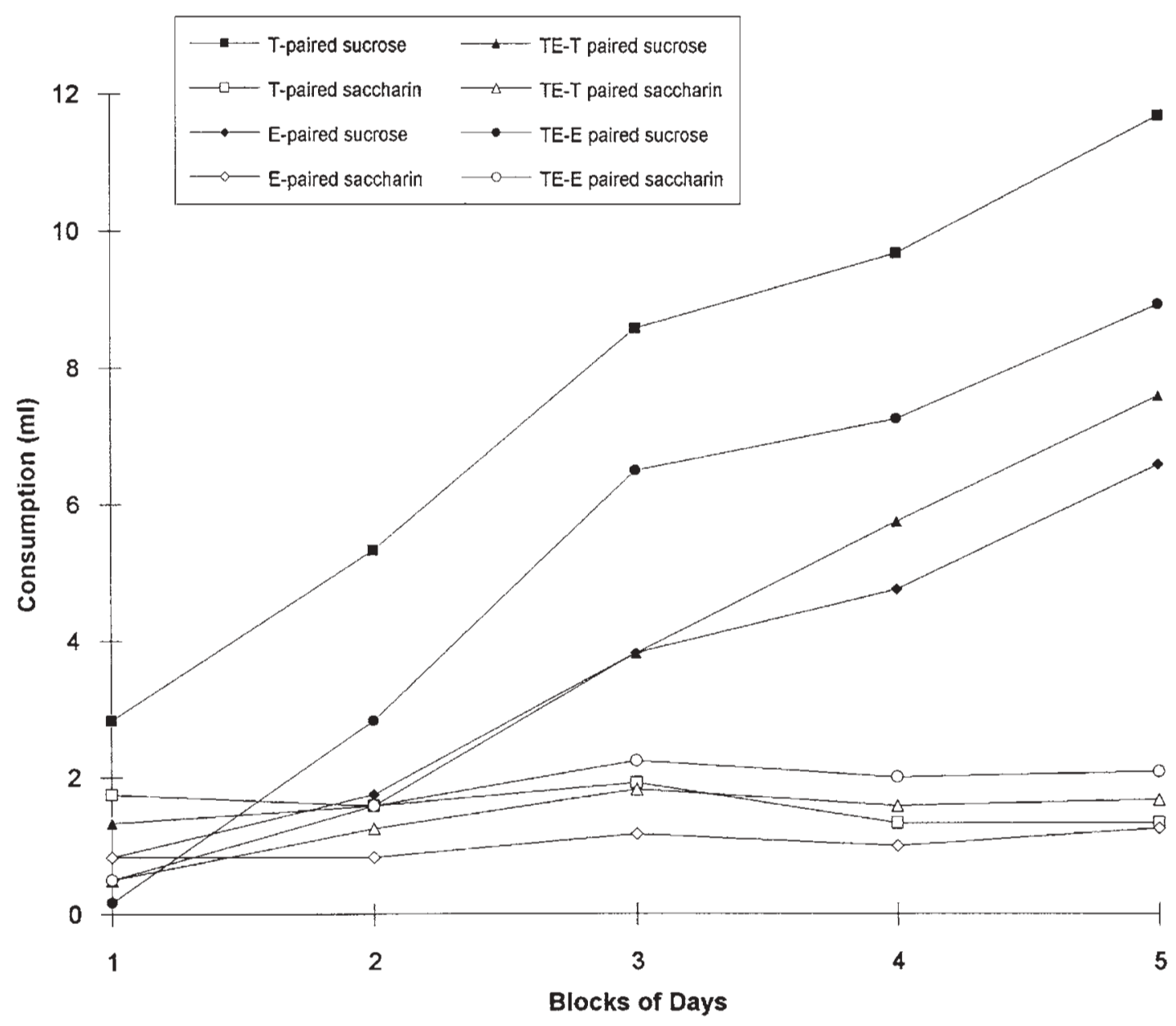

Figure 1. For each group in Experiment 1, mean consumption (in milliliters) of the taste (T), extract (E), or taste/extract compound (TE) mixed with sucrose or saccharin in training. 
group, solution, and days as factors. The difference due to group was significant $[F(3,44)=3.98, p<.02]$. Subsequent Newman-Keuls tests showed that Group T drank significantly more than Groups E and TE-T, and that no other pair of groups differed significantly in amount consumed. The group $\times$ solution interaction was not significant $[F(3,44)=1.71, p>.10]$; Newman-Keuls tests showed that each group drank significantly more of the sucrose solution than of the saccharin solution. The differences due to solution $[F(1,44)=34.72, p<.001]$ and days $[F(4,176)=68.88, p<.001]$ were also significant, as was the solution $\times$ days interaction $[F(4,176)=$ $39.04, p<.001]$. The difference due to solution grew larger over days, as is clear in Figure 1.

Testing. Each group's consumption of the cues (tastes or extracts) that had been paired with sucrose or saccharin is shown in Figure 2. The largest difference in consumption was shown by Group TE-E, and the smallest difference was shown by Group TE-T. That is, when extracts and tastes were conditioned in compound, a larger preference was shown between the extracts than between the tastes. In contrast, when tastes and extracts were conditioned in isolation, a larger preference was shown between the tastes (Group T) than between the extracts (Group E), but the difference was not large.

An ANOVA included group (T, E, TE-E, and TE-T), preference (consumption of the taste or extract that had been paired with sucrose vs. the one that had been paired with saccharin), and days as factors. The difference associated with group was not significant $[F(3,45)=2.29$, $p=.09]$, but the preference was significant $[F(1,45)=$ $12.75, p<.001]$. The group $\times$ preference interaction was not significant $[F(3,45)=2.28, p=.09]$. Consumption decreased from Day 1 to Day 2 of test $[F(1,45)$

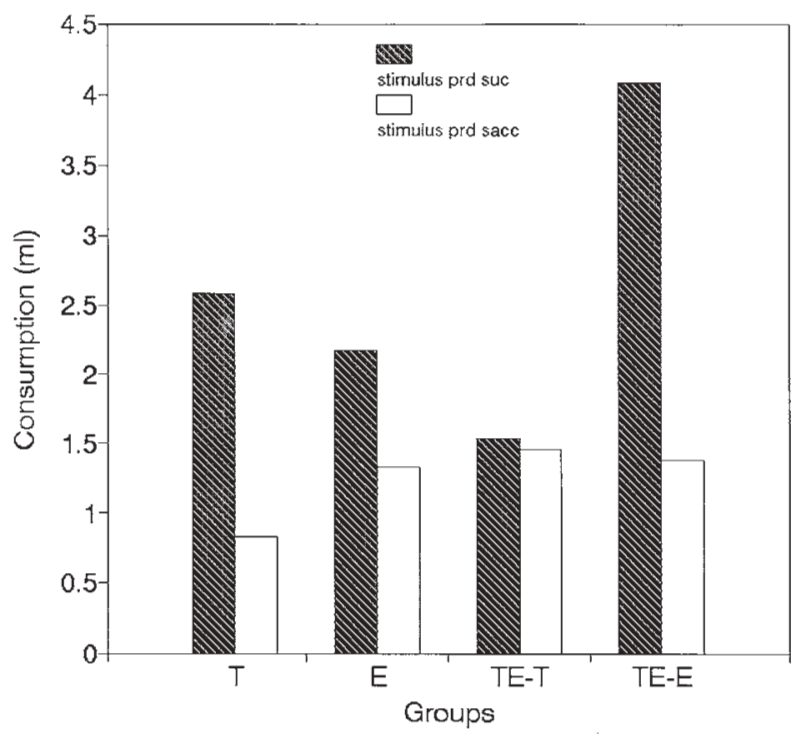

Figure 2. For each group in Experiment 1, mean consumption (in milliliters) of the taste (Groups T and TE-T) or extract (Groups E and TE-E) that had been paired with either sucrose (shaded) or saccharin (unshaded).
$=6.91, p<.02]$, but days did not interact significantly with any other variable.

Because the main aim of the experiment was to determine whether compound conditioning would affect conditioning to the taste and extract elements, size of preference was compared between pairs of groups. Group T's preference was significantly larger than that of Group TE-T $[F(1,20)=7.89, p<.01]$. Group T's preference did not differ significantly from that of Group $\mathrm{E}[F(1,20)=1.31, p>.20]$, and size of preference did not differ significantly for the two groups tested with extracts [TE-E and E; $F(1,20)=1.93, p>.10$ ]. Subsequent Newman-Keuls tests $(p<.05)$ showed that Groups TE-E and T showed a significant preference, but Groups TE-T and E did not.

\section{Discussion}

The effect of taste on conditioning to extract was small in Experiment 1. If anything, taste potentiated conditioning to extract. Group E failed to show a significant preference, but Group TE-E did show a significant preference. When directly compared, however, the preference for Group TE-E was not significantly larger than that for Group E.

It was clear in Experiment 1 that presenting tastes in compound with extracts reduced conditioning to the tastes. This effect was statistically robust-Group TE-T did not show a significant preference, but Group T did, and Group T's preference was significantly larger than that of Group TE-T. This is an overshadowing effect, but the mechanism underlying this effect may be different from that in other cases of overshadowing. Overshadowing refers to interference with the conditioning of a stimulus because of the simultaneous presence of another stimulus that is easier to condition (e.g., Pavlov, 1927). However, in Experiment 1, the extracts were not easier to condition in isolation than the tastes. Indeed, the preference for tastes that had been conditioned in isolation was larger than that for extracts that had been conditioned in isolation, although not significantly so. Thus, the dominance of extracts over tastes when they were presented in compound was apparently not due to the extract's being an inherently more conditionable stimulus than taste.

The results of Experiment 1 cannot be attributed to differences in consumption of sucrose versus saccharin solutions between groups in training. All the groups consumed significantly more of the sucrose solution than of the saccharin solution in Experiment 1, and they did not differ significantly in the size of this difference.

The results of Experiment 1 contrast with the typical results in taste-aversion conditioning, which have shown that tastes potentiate conditioning to extracts and extracts have no effect on conditioning to tastes (e.g., Palmerino et al., 1980). Our design was more complex than that usually used in aversion conditioning. In training, two tastes, two odors, sucrose, and saccharin were used. The use of two tastes and two odors is necessary if a two-bottle preference test is to be used. It has been 
shown that one-bottle tests are less sensitive to preference than two-bottle tests, and Sclafani (1987) has suggested that the one-bottle test might be better termed "acceptance" rather than "preference." The procedure of mixing the cues in with the reinforcers (sucrose and saccharin) has been used successfully in the past to produce conditioned preferences. Conditioned preferences have also been obtained when the cues precede the reinforcers (e.g., Capaldi et al., 1987; Holder, 1991). Mixing the cues in with the reinforcers does not interfere with conditioning preferences, but instead seems to actually facilitate conditioning (e.g., Boakes, Rossi-Arnaud, \& Garcia-Hoz, 1987). However, perhaps in our taste/extract compound groups, an additional taste could not potentiate conditioning to extract because the taste of sucrose and saccharin already potentiated conditioning. However, this account implies that conditioning in Group E should have been potentiated by the tastes of sucrose and saccharin. Yet in Experiment 1, Group E failed to show a significant preference.

In conditioned preference learning, other procedures are necessarily different from those used in aversion learning. For example, the number of conditioning trials is small in aversion learning, and water deprivation is usually used instead of food deprivation, among many other differences. At the present time, we are directly comparing aversion and preference conditioning to tastes and extracts in our laboratory by using common procedures.

Here, we pursued the role of taste and extract in flavor preference conditioning. The largest effect in Experiment 1 was the overshadowing of taste by extract. Group $\mathrm{T}$ showed a significant preference, but Group TE$\mathrm{T}$ did not. One interpretation of overshadowing is in terms of competition between CSs for associative strength (e.g., Mackintosh, 1975; Rescorla \& Wagner, 1972). The idea is that the most salient stimulus will garner most of the associative strength, overshadowing the weaker stimulus. This would imply that Group TE-T failed to show a significant preference because the extract stimulus garnered most of the associative strength. An argument against this interpretation can be shown by the fact that, in Experiment 1, when tastes and extracts were conditioned in isolation (Groups T and E), Group T showed a significant preference, while Group E did not. However, perhaps the results of conditioning stimuli in isolation is not a good measure of the salience of those same stimuli presented in compound. Perhaps when tastes and extracts are combined, the tastes are less salient than the extracts, even though in isolation tastes and extracts are both salient enough to produce strong conditioning.

In Experiment 2, we conditioned tastes in isolation prior to training with a taste/extract compound. The idea was that conditioning tastes in isolation would produce an associative strength to the tastes that would be maintained when the tastes were combined with the extracts. Accordingly, a group trained with a taste/extract compound following training with taste alone might show a significant conditioned preference to the taste cue. In addition, prior conditioning to tastes could actually interfere with conditioning to extracts when the two stimuli were compounded - that is, conditioning of tastes might "block" conditioning to extracts. Blocking refers to the reduced conditioning obtained when a stimulus is presented in compound with a previously conditioned stimulus (e.g., Kamin, 1969).

\section{EXPERIMENT 2}

In Experiment 2, we used the same taste and extract stimuli that were used in Experiment 1, and we used procedures devised by Holder (1991) to measure blocking in conditioned flavor preference learning. In the first phase of his experiment, Holder paired one odor or taste with sucrose and a second odor or taste with saccharin. In Holder's Phase 2, the other components (odor or taste) were paired with sucrose or saccharin. For half of the rats (Group Same), the assignment of flavor component to sucrose or saccharin was the same in the two phases. For example, in Phase 1, if almond was paired with sucrose, and peppermint was paired with saccharin, this was also true in Phase 2; in addition, citric acid was added to the almond-sucrose, and salt was added to the peppermint-saccharin. For the other half of the rats (Group Different), the pairings were reversed from Phase 1 to Phase 2. So if almond was paired with sucrose, and peppermint was paired with saccharin in Phase 1, in Phase 2, almond was paired with saccharin, and peppermint was paired with sucrose; in addition, citric acid was added to the almond-saccharin, and salt was added to the peppermint-sucrose. Blocking was indicated by a weaker preference between the stimuli added in Phase 2 for Group Same than for Group Different (Holder, 1991).

In Experiment 2, the rats were trained with tastes in Phase 1, and the extracts were added to the tastes in Phase 2. We used Holder's (1991) design with the addition of a control group that was not trained in Phase 1; we also gave tests with both extracts and tastes (Holder tested only with the stimulus added in Phase 2). Also, we used extracts in water as odorous stimuli, whereas Holder used odor stimuli presented on disks around the water spout, and we trained under food deprivation rather than under water deprivation.

\section{Method}

Subjects. The subjects were 30 naive, male Sprague-Dawley rats from Charles River, Inc., Wilmington, MA. They were 78 days old upon arrival and 81 days old at the start of the experiment.

Materials and Apparatus. These were the same as those used in Experiment 1.

Procedure. Water was present ad lib in clear plastic bottles mounted to the right of the food hopper on each cage front. On Day 1, all food was removed from the cages. On Days 2-11, the rats were fed $14 \mathrm{~g}$ each day at 9:00 a.m. During training at 10:30 a.m., they were fed at 1:00 p.m. Phase 1 of training began on Day 12 and lasted for 10 days. The rats were randomly divided into three groups. Group Control simply rested in their cages during this phase. Groups Same and Different were treated identically in Phase 1; they received Taste 1 paired with sucrose and Taste 2 
paired with saccharin. For half of the rats in each group, Taste 1 was salt and Taste 2 was citric acid; for the other half, the reverse was true. The rats received 10 -min access to $40 \mathrm{ml}$ of one solution each day. On half of the days, the sucrose solution was given; on the other days the saccharin solution was given, with the order of the solutions double alternating — half the rats began with sucrose and half began with saccharin.

Phase 2 began the day after Phase 1 ended, and it lasted for 10 days. In Phase 2, Group Same received the same tastes paired with sucrose and saccharin that they had in Phase 1, but extracts were added. In Phase 2, Group Different received pairings of tastes with sucrose and saccharin that were reversed from those in Phase 1, but extracts were added. Taste 1 , which had been added to sucrose in Phase 1, was added to saccharin plus an extract in Phase 2; Taste 2, which had been added to saccharin in Phase 1, was added to sucrose plus an extract in Phase 2. Group Control received one taste/extract combination with sucrose and another with saccharin. For half of the rats in each group, peppermint was paired with sucrose, and almond was paired with saccharin; the other half received peppermint with saccharin and almond with sucrose.

The extract test began the day after Phase 2 ended. In the extract test, all the groups received a 4-h two-bottle test between $40 \mathrm{ml}$ of peppermint-flavored water and $40 \mathrm{ml}$ of almond-flavored water (normal water bottle was removed). There were 2 days of test; bottle positions were reversed between Days 1 and 2. The taste test began the day after the extract test ended. In the taste test, all the groups received a 4-h two-bottle test between $40 \mathrm{ml}$ of citric acid in water and $40 \mathrm{ml}$ of salt in water. There were 2 days of taste testing; bottle positions were reversed between Days 1 and 2 .

\section{Results}

Training. Figure 3 shows the consumption of sucrose and saccharin in both Phases 1 and 2 of training for each group. As can be seen in the figure, in Phase 1, Group Same and Group Different both drank more sucrose than saccharin, and consumption of sucrose increased over days. An ANOVA showed that the consumption of sucrose was greater than that of saccharin $[F(1,18)=$ 255.63, $p<.001]$, and that this difference increased over days $[F(4,72)=63.18, p<.001]$. There were no interactions involving group $\left(F_{\mathrm{S}}<1\right)$.

As Figure 3 also shows, introduction of the extract in Training Phase 2 reduced consumption of sucrose for Groups Same and Different. Over the days of Phase 2, consumption of sucrose increased for all the groups. An ANOVA on the consumption data of Training Phase 2 showed that consumption of sucrose was greater than that of saccharin $[F(1,27)=22.95, p<.001]$; this difference did not vary by group $(F<1)$. The difference between sucrose and saccharin grew larger over days $[F(4,108)=31.00, p<.001]$, and this did not vary by group $(F=1.01)$.

Testing. In the extract test, all the rats were given a choice between almond and peppermint - the extracts that were added to the solutions in Phase 2. Blocking would be indicated by a smaller consumption difference between the extract paired with sucrose and that paired with saccharin for Group Same than for Group Different and Group Control (i.e., a smaller conditioned preference for Group Same). Figure 4 shows the mean consumption of the extract that had been paired with sucrose and the extract that had been paired with saccharin for each group. Each group preferred the extract that had been paired with sucrose, and this difference was indeed smallest for Group Same. However, the differences between groups in size of preference were not significant. An ANOVA included group (same, different, control), extract (paired with sucrose vs. paired with

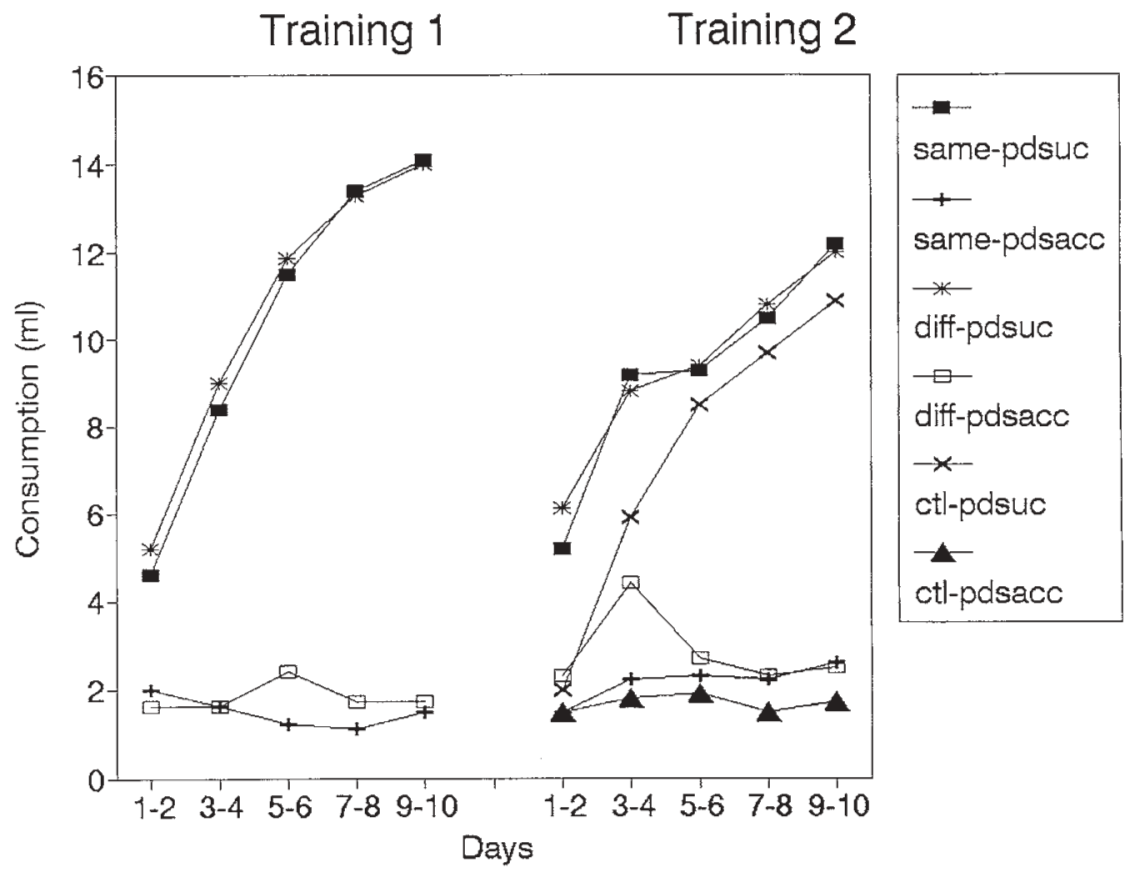

Figure 3. For each group in Experiment 2, mean consumption (in milliliters) of the stimuli paired with sucrose (pdsuc) or saccharin (pdsacc) in Phases 1 and 2 of training. 


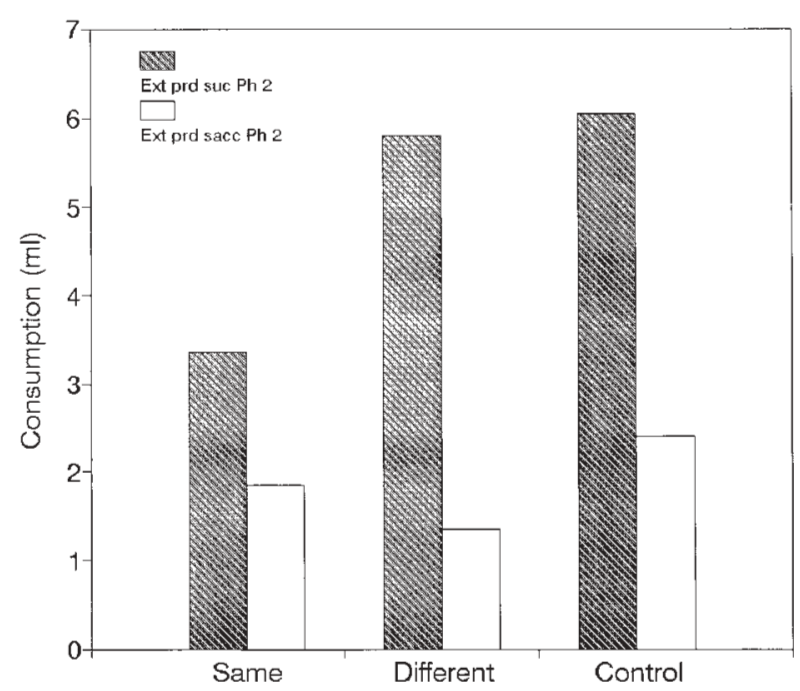

Figure 4. For each group in Experiment 2, mean consumption (in milliliters) of the extract that had been paired with sucrose (shaded) in Phase 2 versus the extract that had been paired with saccharin (unshaded) in Phase 2 (Test Phase 1).

saccharin), and days as factors. The differences due to group $[F(1,27)=63.69, p<.001]$ and extract $[F(1,27)$ $=13.14, p<.01]$ were significant, but the group $\times$ extract interaction was not $[F(2,27)=1.0, p>.30]$. This indicates that the preference between the extracts did not vary significantly by group. There were also no significant group $\times$ extract interactions when pairs of groups were compared [Group Same vs. Group Control, $F<1$; Group Same vs. Group Different, $F(1,18)=2.36]$. Consumption decreased from Day 1 to Day 2 of test $[F(1,27)=16.01, p<.001]$. This decrease did not interact with any other factor.

Figure 5 shows Test 2 results - the mean amount consumed by each group of the taste that had been paired with sucrose and of the taste that had been paired with saccharin in Training Phase 2. As can be seen in the figure, Group Control showed no preference between the two tastes, but Group Same preferred the taste that had been paired with sucrose in both training phases. Most interesting is the performance of Group Different- they preferred the taste that had been paired with sucrose in Training Phase 1 (and saccharin in Phase 2), and not the taste that had been paired with sucrose in Training Phase 2.

An ANOVA included group (same, different, control), taste (paired with sucrose in Phase 2 vs. paired with saccharin in Phase 2), and days as factors. The difference due to group was not significant $(F<1)$, nor was the difference due to taste $(F<1)$. However, the group $\times$ taste interaction was significant $[F(2,27)=7.88, p<$ .01], reflecting the opposite preferences of Group Different and Group Same and the lack of preference for Group Control. Subsequent Newman-Keuls tests showed that the preferences for Group Same and Group Different were significant, but preference for Group Control was not. An analysis comparing Groups Different and Same showed a significant group $\times$ taste interaction $[F(1,18)=14.9, p<.01]$. Consumption decreased from Day 1 to Day 2 of test $[F(1,27)=8.11, p<.01]$. This decrease did not interact with any other factor.

\section{Discussion}

Extracts overshadowed conditioning to tastes in Phase 2 of Experiment 2. Group Control, trained only with the taste/extract compound, showed a significant preference between the extracts, but no preference between the tastes. Pretraining the tastes did not affect conditioning to the taste/extract compound: Group Same showed the same preferences as Group Control. Group Different, which was trained with the tastes in isolation in Phase 1, showed a significant preference between the tastes consistent with their Phase 1 training. Thus, both Experiments 1 and 2 show that tastes are conditionable when presented in isolation. It is only when tastes are presented in compound with extracts that no conditioned preference to taste is shown. The taste cue is changed somehow by being presented in compound with the extract.

Pearce (1987), for one, suggested that subjects respond to stimuli in compound in terms of the unique configuration of the stimulus elements. In his view, overshadowing reflects a greater similarity between the compound stimulus and one of the stimulus elements. Applying this analysis to results of Experiments 1 and 2 , the overshadowing of taste by extract indicates that the taste/extract compound is more similar to the extract than to the taste.

The results for Group Different are consistent with this interpretation; they showed a significant preference between tastes consistent with the conditioning the tastes received in Phase 1 when presented in isolation.

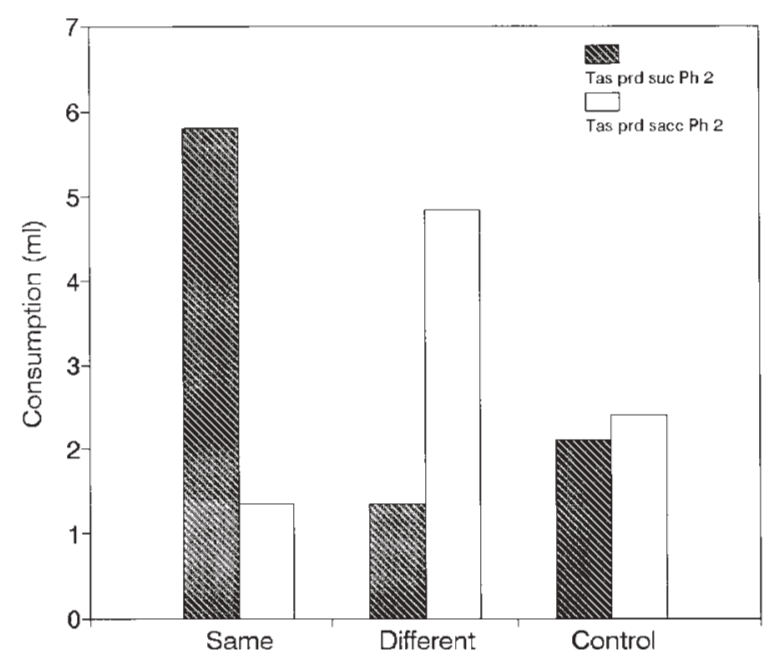

Figure 5. For each group in Experiment 2, mean consumption (in milliliters) of the taste that had been paired with sucrose (shaded) in Phase 2 versus the taste that had been paired with saccharin (unshaded) in Phase 2 (Test Phase 2). 
That is, Group Different's taste preference showed that the tastes were still associated with the solutions from Phase 1, despite the fact that the tastes received reverse pairings in compound with extracts in Phase 2. This is consistent with the idea that the taste/extract compound produces a configuration that is more similar to the extract in isolation than to the taste in isolation. The taste stimuli maintained their original difference in associative strength because, functionally, the taste stimuli were not present in Phase 2. This interpretation explains why conditioned preferences can be formed to tastes in isolation, but conditioning of a taste/extract compound does not generalize to tastes - a finding that is difficult to interpret in terms of salience.

If the taste/extract compound is similar to the extract in isolation, as suggested by the results of Experiments 1 and 2, conditioning accruing to an extract stimulus will be affected by subsequent conditioning given to a taste/ extract compound. This is because the extract apparently maintains its identity in the taste/extract compound. Thus, if an extract is paired with (preferred) sucrose preceding the pairing of a taste/extract compound with (less preferred) saccharin, the extract should become associated with saccharin rather than sucrose.

In actuality, training must include two extracts in order to measure a preference in test. In Experiment 3, Group Different received Extract 1 paired with sucrose and Extract 2 paired with saccharin in Phase 1. In Phase 2, the extracts were combined with tastes and the contingencies were reversed: Extract 1(+ taste) was paired with saccharin, and Extract 2(+ taste) was paired with sucrose. We expected that preference in a later test would reflect Phase 2 training, not Phase 1 training.

\section{EXPERIMENT 3}

In Experiment 3, we used the same design that we had used in Experiment 2, but training was given with the extracts in Phase 1, and tastes were added to the extracts in Phase 2.

\section{Method}

Subjects. The subjects were 30 naive male rats of the same description as those employed in Experiments 1 and 2. They were 77 days old on arrival and 80 days old at the start of the experiment.

Materials and Apparatus. These were the same as those used in Experiments 1 and 2.

Procedure. The procedure was identical to that in Experiment 2, except that in Phase 1, Groups Same and Different were trained with extracts (almond and peppermint); in Phase 2, tastes (salt and citric acid) were added to the extracts. In the first test phase, the rats were tested with tastes, and in the second test phase they were tested with extracts.

\section{Results}

Training. Figure 6 shows consumption (in milliliters) of saccharin and sucrose for each group in Training Phases 1 and 2.

In Training Phase 1, Groups Same and Different both drank more sucrose than saccharin $[F(1,18)=16.03$, $p<.001]$. This difference did not vary by group $(F<1)$.
Consumption increased over days $[F(4,15)=11.38, p<$ $.001]$, due to an increase in sucrose consumption [sucrose vs. saccharin $\times$ days, $F(4,72)=22.68, p<.001]$. This effect did not vary by group $(F<1)$. As Figure 6 shows, adding a taste to the extract for Group Same produced no change in sucrose or saccharin consumption. Recall that, in Experiment 1, adding extracts to tastes produced a change in sucrose and saccharin consumption for Group Same. This aspect of the results is consistent with the idea that the taste/extract combination is more discriminable from taste alone than from extract alone. Adding extracts to tastes (Experiment 1) produced more of a change in consumption than adding tastes to extracts (Experiment 2).

Reversing the extracts and adding a taste for Group Different in Training Phase 2 produced both a drop in sucrose consumption and an increase in saccharin consumption. Over days of Training Phase 2, consumption for Group Different and Group Control increased more than that for Group Same. This pattern of results in Training Phase 2 produced a significant effect of days $[F(4,108)=26.87, p<.001]$ and a significant days $\times$ groups interaction $[F(8,108)=6.39, p<.001]$. In Training Phase 2, all the groups consumed more of the sucrose solution than of the saccharin solution $[F(1,27)=19.76$, $p<.001]$; this difference did not vary by group $(F<1)$.

Testing. In Test Phase 1, all the rats were given a choice between salt and citric acid - the tastes that were added to the solutions in Phase 2. Figure 7 shows the mean consumption of the taste that had been paired with sucrose and the taste that had been paired with saccharin for each group. As can be seen in Figure 7, Groups Same and Control showed no preference, whereas Group Different had a small preference for the taste that had been paired with sucrose. However, there was no significant effect of taste (paired with sucrose vs. paired with saccharin; $F<1)$ or group $\times$ taste interaction $[F(2,27)=$ $1.22, p>.30]$. The effect of group was also not significant $(F<1)$. Consumption decreased from Test Day 1 to Test Day $2[F(1,27)=8.53, p<.01]$. This decrease did not interact with any other factor.

In Test Phase 2, all the groups were tested with the extracts. Figure 8 shows the mean consumption of the extracts that were paired with sucrose or saccharin in Phase 2 for each group. As can be seen in Figure 8, all the groups preferred the extract that had been paired with sucrose in Phase 2-even Group Different, for which extract pairings with sucrose and saccharin were reversed from Training Phases 1 to 2 . The difference due to extract (paired with sucrose vs. paired with saccharin) was significant $[F(1,27)=19.40, p<.001]$. There were no significant differences associated with group $(F<1)$ or group $\times$ extract $(F<1)$. Consumption decreased from Day 1 to Day 2 of test $[F(1,27)=62.6, p<.001]$. This decrease did not interact with any other factor.

\section{Discussion}

In Experiment 3, conditioning to extracts had no effect on subsequent conditioning involving a taste/extract 


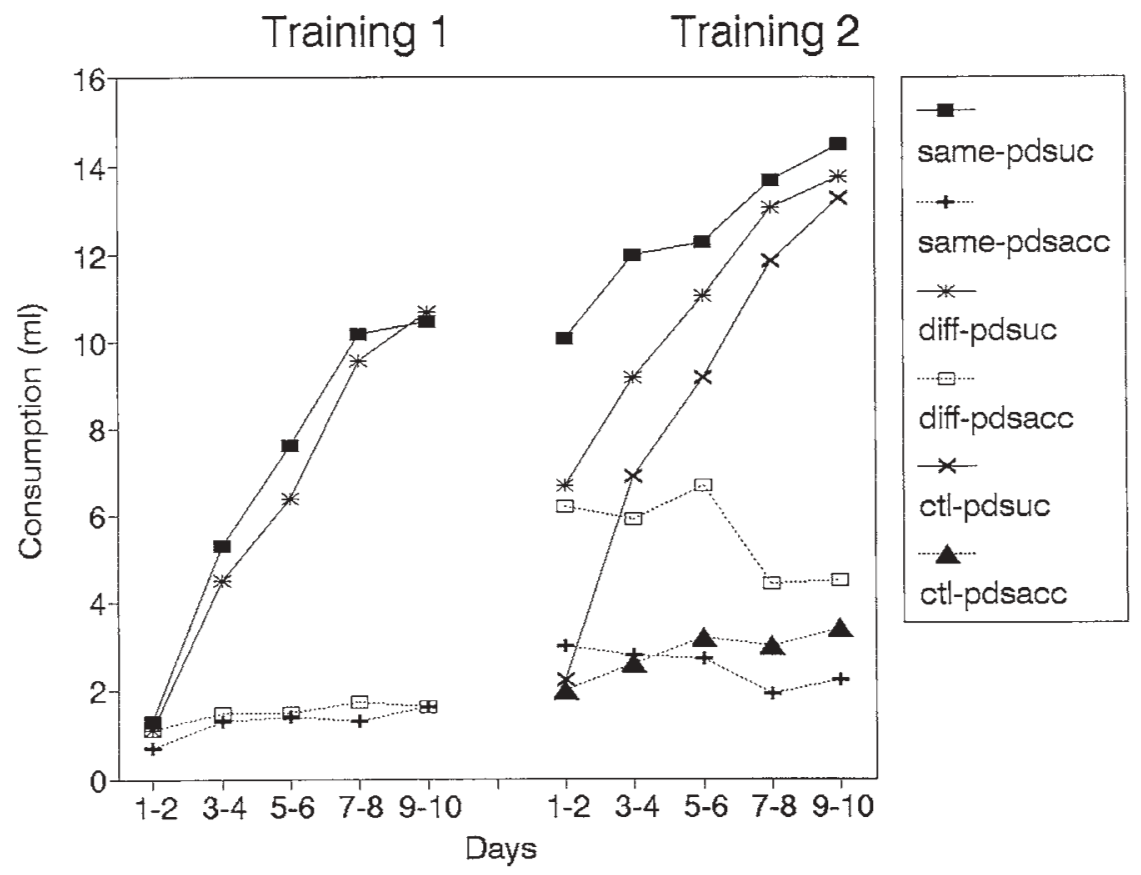

Figure 6. For each group in Experiment 3, mean consumption (in milliliters) of the stimuli paired with sucrose (pdsuc) or saccharin (pdsacc) in Phases 1 and 2 of training.

compound. Conditioning of the taste/extract compound produced a preference for the extract paired with sucrose in Phase 2 and no preference for the taste paired with sucrose in Phase 2, regardless of previous conditioning history. As expected, Group Different preferred the extract paired with sucrose in Phase 2-not the extract paired with sucrose in Phase 1. This result shows that the extract stimulus in the taste/extract compound is similar to the extract stimulus when presented alone. Thus, re-

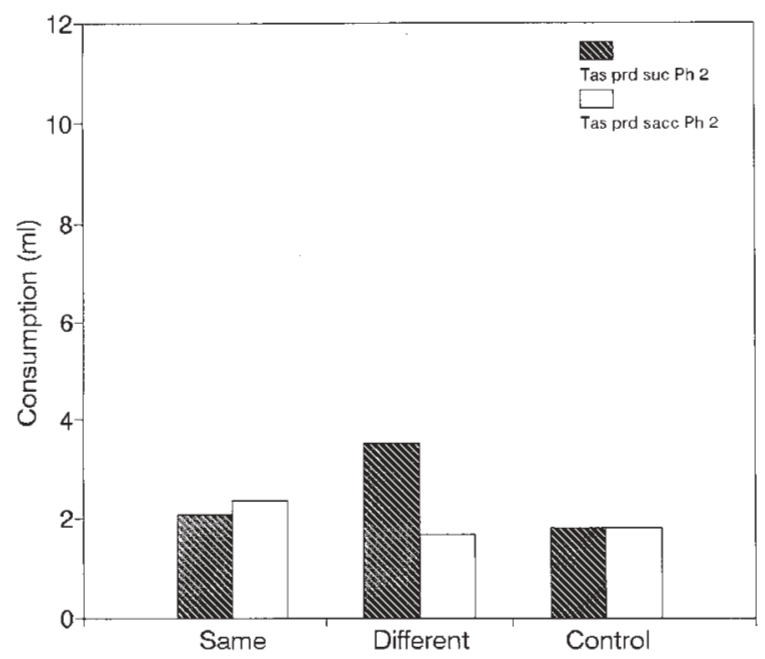

Figure 7. For each group in Experiment 3, mean consumption (in milliliters) of the taste that had been paired with sucrose (shaded) in Phase 2 versus the taste that had been paired with saccharin (unshaded) in Phase 2 (Test Phase 1). versing the contingencies for extract between extractalone training in Phase 1 and taste/extract training in Phase 2 reversed the preferences shown when the rats were tested with extracts alone.

None of the groups in Experiment 3 showed a significant taste preference, because tastes were only experienced in compound with extracts in Experiment 3. In Experiment 2, preferences were shown for the taste associated with sucrose in Phase 1, when taste was presented independently for conditioning. Thus, conditioned preferences can be formed to tastes when tastes are presented in isolation. However, when tastes are presented only in compound with extracts and never alone, conditioning does not generalize to the taste that is presented in isolation.

\section{EXPERIMENT 4}

The previous experiments have shown that tastes are conditionable in isolation, but not in compound with extracts. One relatively uninteresting explanation of the results of these experiments is that, for some reason, the rats could not taste citric acid and salt in the presence of almond and peppermint. Of course, this may indicate overshadowing, in which the presence of one stimulus interferes with sensing another stimulus. Thus, perhaps extracts do interfere with tasting salt and citric acid. Alternatively, the rats may taste the stimuli, but presenting tastes with extracts may change the taste stimuli so that they are not similar to the taste stimuli presented in isolation. In Experiment 4, we asked whether rats could learn a discrimination that required them to taste salt in 


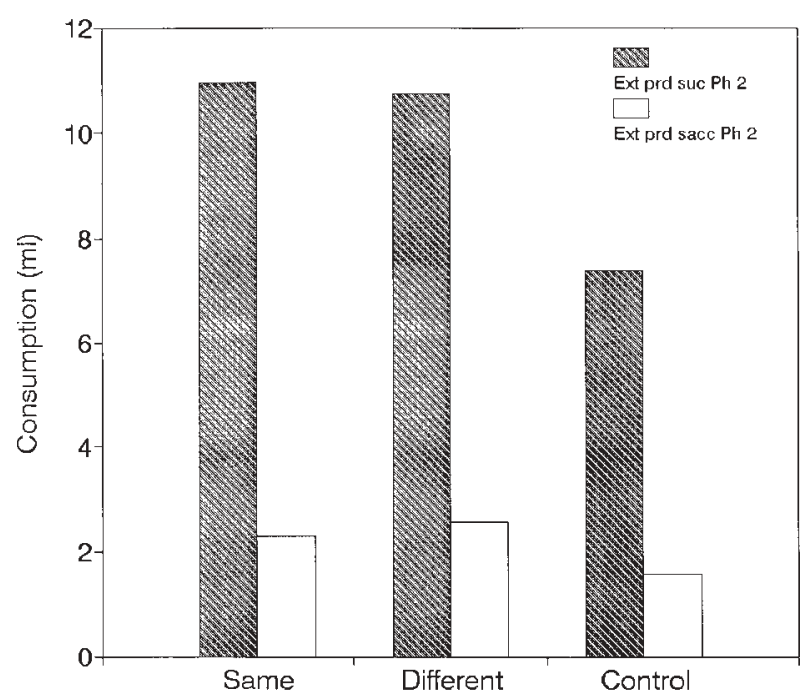

Figure 8. For each group in Experiment 3, mean consumption (in milliliters) of the extract that had been paired with sucrose (shaded) in Phase 2 versus the extract that had been paired with saccharin (unshaded) in Phase 2 (Test Phase 2).

compound with almond extract. They received three types of trials, each six times: almond-sucrose, saltsucrose, and almond-salt-saccharin. This is a configural conditioning design of the negative-patterning type (e.g., Kehoe \& Gormezano, 1980). The compound stimulus is associated with saccharin $(\mathrm{S}-)$, but each element is associated with sucrose $(\mathrm{S}+)$. After this training, the rats were tested with almond+salt versus almond, or almond+salt versus salt. If almond-salt produces a unique configuration, the animals should prefer almond alone or salt alone to this configuration. If the rats cannot taste salt in the presence of almond extract, the training becomes, functionally, almond-sucrose, almondsaccharin, and salt-sucrose, each six times, and testing is with almond versus almond, and almond versus salt. Rats that cannot taste salt in the presence of almond should show a preference for salt when tested with salt versus almond + salt (functionally almond), but no preference when tested with almond + salt (functionally almond) versus almond.

\section{Method}

Subjects. The subjects were 12 naive rats with the same characteristics as those in the previous experiments. They were 75 days old upon arrival and 78 days old at the start of the experiment.

Materials and Apparatus. These were the same as those used in the previous experiments, except only salt and almond were used.

Procedure. Upon arrival, the rats rested undisturbed for 3 days before they were placed on a $14 \mathrm{~g}$ per day food-deprivation schedule for 10 days, which lasted throughout the experiment. During the 18 days of training, the rats received the following three solutions for 6 days each: salt + sucrose, almond + sucrose, and almond + salt + saccharin. On each training day, $40 \mathrm{ml}$ of one of these solutions was given for $10 \mathrm{~min}$. The order of presentation was randomized.
In two-bottle testing, half of the rats were tested between salt and almond + salt, and the other half were tested between almond and almond + salt. Forty milliliters of each solution were given in test for $4 \mathrm{~h}$ each day. There were 4 days of testing.

\section{Results}

Figure 9 shows the mean consumption of almond (A) and salt (S) when each was paired with sucrose in training. Also shown is consumption of the almond-salt compound $(\mathrm{A} / \mathrm{S})$, which was paired with saccharin. As can be seen in Figure 9, there was greater consumption of the stimuli paired with sucrose than of the stimulus paired with saccharin. An ANOVA showed a significant difference in consumption on the three types of day [salt, almond, almond-salt; $F(2,10)=315.04, p<.001]$. Subsequent Newman-Keuls tests showed that the difference between each solution was significant; the rats consumed more salt-sucrose than almond-sucrose, and more almond-sucrose than almond-salt-saccharin.

Figure 10 shows the mean consumption of almond (A) versus almond + salt $(\mathrm{A} / \mathrm{S})$, and salt $(\mathrm{S})$ versus almond + salt $(A / S)$ in testing. As can be seen in the figure, the rats consumed more of the stimulus that had been paired with sucrose, whether it was almond or salt, than of the compound stimulus almond + salt. This difference was significant $[F(1,10)=14.87, p<.01]$ and did not vary with whether almond or salt was the single stimulus $(F<1)$.

\section{Discussion}

If the rats could not taste salt in compound with almond, it would be impossible for them to learn that almond and almond + salt were associated with two different solutions. However, the rats preferred almond to almond + salt, showing that they had learned that these two stimuli were associated with different solutions and that they could taste the salt in compound with the almond.

The design of Experiment 4 was a negative-patterning design (Kehoe \& Gormezano, 1980), which is a test of whether a unique configural cue is produced by a compound stimulus, rather than the compound merely representing the two elements. The fact that the rats formed a conditioned preference for either element versus the compound stimulus suggests that a unique configural cue is produced by the compound stimulus almond+ salt. If this were not true, and the compound stimulus almond + salt were identical to the sum of almond alone and salt alone, the problem presented in Experiment 4 would be unsolvable. Almond and salt were both paired six times with sucrose and six times with saccharin. Thus, if the almond + salt compound did not produce a unique cue, there would be no basis for preference in the test.

The results of Experiments 1, 2, and 3 suggest that the taste/extract compound stimulus is more similar to the extract presented alone than to the taste presented alone. Experiment 4 shows that, despite this, animals can learn a discrimination between an extract and a taste/extract compound, indicating that the taste contributes to the 


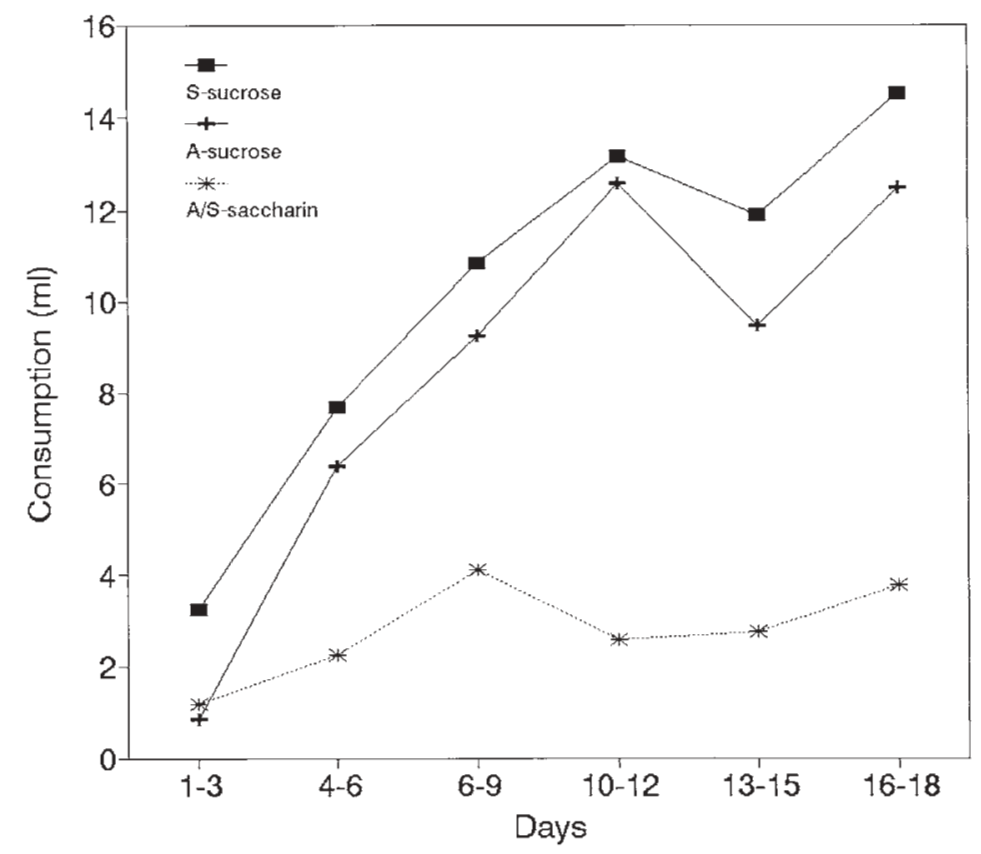

Figure 9. Mean consumption (in milliliters) of the salt (S)-sucrose, almond (A)sucrose, and almond/salt (A/S)-saccharin solutions in Experiment 4.

compound configuration. Indeed, when specific training is given on a discrimination problem between taste alone or extract alone versus the taste + extract compound, as was done in Experiment 4, discrimination performance is as good to taste versus taste + extract as it is to extract versus taste + extract. This is consistent with many studies showing that explicit discrimination train-

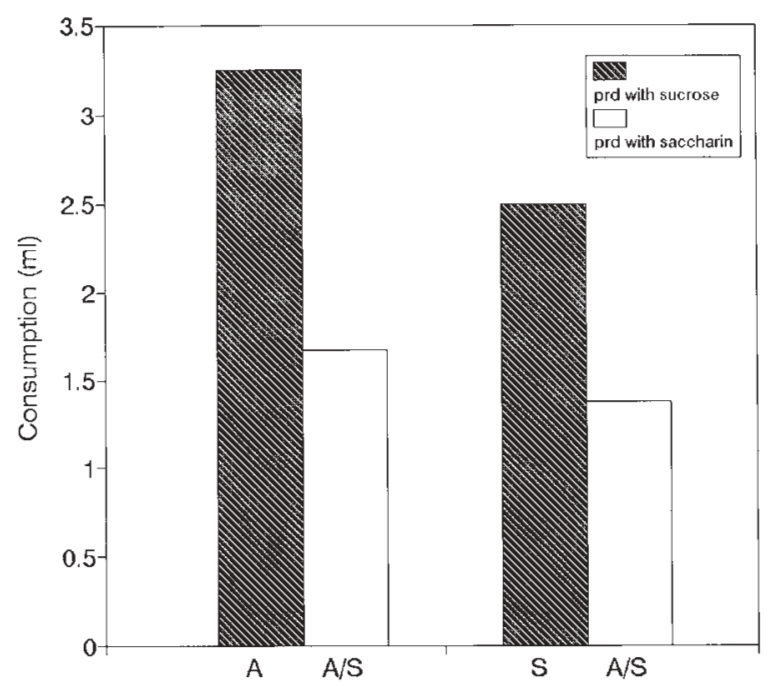

Figure 10. Mean consumption (in milliliters) of the test between almond (A) paired with sucrose and almond/salt (A/S) paired with saccharin, and between salt (S) paired with sucrose and almond/salt paired with saccharin in Experiment 4. ing sharpens generalization gradients (e.g., Terrace, 1966).

\section{GENERAL DISCUSSION}

We found here that extracts overshadowed conditioning to tastes (Experiment 1) in flavor preference learning: When tastes and extracts were presented in compound, preference was shown between the extracts, but not the tastes. The presence of extracts prevented conditioning to tastes in all three experiments. This did not occur because the tastes were ineffective stimuli. When tastes were conditioned independently prior to compound conditioning (Experiment 2), conditioned preferences were formed to the tastes. Moreover, Experiment 4 showed that the rats could taste salt when it was in compound with almond.

Experiment 4 also showed that rats could form a preference for a taste and extract presented separately versus the same taste and extract in compound. This finding suggests that the taste/extract compound produces a unique cue that is not possessed by the taste and extract stimuli conditioned in isolation.

The results of all four experiments taken together support a configural view of compound stimuli and overshadowing (e.g., Pearce, 1987), as opposed to a stimuluselements view that attributes overshadowing to competition between the stimulus elements. The following is a summary of the main findings in support of this conclusion. First, the taste cue does not seem to be inherently weaker than the extract cue. When conditioned independently, both taste and extract were conditionable (Experiment 1 ), and neither was significantly stronger than 
the other. Experiment 2 also showed that conditioned preferences could be formed to taste cues presented in isolation. Second, Experiments 2 and 3 both showed that when a taste/extract compound was conditioned, conditioning did not generalize to the taste cue presented alone, but did generalize to the extract cue presented alone. This finding indicates that the taste/extract compound changes the nature of the taste cue more than it changes the extract cue. Third, Experiment 4 showed that the extract cue did not prevent sensing of the taste cue; rather, the taste/extract compound produces a unique cue not possessed by the elements of taste and extract. Because conditioning of the taste/extract compound generalizes to extract alone, but not taste alone, the nature of the unique cue produced by the compound is a change in the taste element that is bigger than any change in the extract element. Fourth, conditioning of a taste/extract compound did not counteract prior conditioning to taste (Experiment 2), presumably because the taste element is changed by being presented in compound with an extract. In contrast, conditioning to the extract element in isolation was counteracted by later conditioning of the taste/extract compound (Experiment 3). Thus, the extract element must be present in relatively unchanged form in the taste/extract compound.

These experiments show that taste and extract interact in unusual ways in conditioned flavor preference conditioning. It is too simplistic to say that extract is more salient than taste. Rather, it appears that both stimuli are equally salient. However, when presented in compound, a unique configural stimulus that is more similar to the extract than to the taste is produced. Because taste and extract change when they are combined, blocking failed to occur when either taste or extract was conditioned prior to conditioning the taste/extract compound. Blocking requires that the stimulus element conditioned in Phase 1 be present in Phase 2. We are suggesting that in the case of taste, presence of the extract changes the taste cue, so that the taste cue is functionally not present in Phase 2 . The change in the taste cue prevents prior conditioning to taste from interfering with conditioning to extract. Prior conditioning to extract could not block conditioning to taste in the taste/extract compound for a different reason. Conditioning of the taste/extract compound does not generalize to taste in isolation. Since the control group showed no conditioned preference to taste after conditioning of a taste/extract compound, there is no room to measure blocking of conditioning to taste by prior conditioning of the extract.

We attribute the present results to characteristics of the stimulus produced when tastes and extracts are combined. It remains to be determined why combining tastes and extracts in flavor preference conditioning produces different results than combining tastes and extracts in flavor-aversion learning. This difference between aversion and preference conditioning is unanticipated by current theory.

\section{REFERENCES}

Boakes, R. A., Rossi-Arnaud, C., \& Garcia-Hoz, V. (1987). Early experience and reinforcer quality in delayed flavour-food learning in the rat. Appetite, 9, 191-206.

Bouton, M. E., \& Whiting, M. R. (1982). Simultaneous odor-taste and taste-taste compounds in poison-avoidance learning. Learning \& Motivation, 13, 472-494.

Capaldi, E. D., Campbell, D. H., Sheffer, J. D., \& Bradford, J. P. (1987). Conditioned flavor preferences based on delayed caloric consequences. Journal of Experimental Psychology, 13, 150-155.

Durlach, P. J., \& Rescorla, R. A. (1980). Potentiation rather than overshadowing in flavor-aversion learning: An analysis in terms of within-compound associations. Journal of Experimental Psychology: Animal Behavior Processes, 6, 175-187.

Garcia, J. (1989). Food for Tolman: Cognition and cathexis in concert. In T. Archer \& L. Nilsson (Eds.), Aversion, avoidance, and anxiety (pp. 45-85). Hillsdale, NJ: Erlbaum.

Hankins, W. G., Garcia, J., \& Rusiniak, K. W. (1973). Dissociation of odor and taste in baitshyness. Behavioral Biology, 8, 407-419.

Holder, M. D. (1991). Conditioned preferences for the taste and odor components of flavors: Blocking but not overshadowing. Appetite, 17, 29-45.

Holman, E. W. (1975). Immediate and delayed reinforcers for flavor preferences in rats. Learning \& Motivation, 6, 91-100.

Kamin, L. J. (1969). Predictability, surprise, attention, and conditioning. In B. A. Campbell \& R. M. Church (Eds.), Punishment and aversive behavior (pp. 279-296). New York: Appleton-CenturyCrofts.

Kehoe, E. J., \& Gormezano, I. (1980). Configuration and combination laws in conditioning with compound stimuli. Psychological Bulletin, 87, 351-378.

Mackintosh, N. J. (1975). A theory of attention: Variations in the associability of stimuli with reinforcement. Psychological Review, 82 276-298.

Mehiel, R., \& Bolles, R. C. (1984). Learned flavor preferences based on caloric outcome. Animal Learning \& Behavior, 12, 421-427.

Mikulka, P. J., Pitts, E., \& Philput, C. (1982). Overshadowing not potentiation in taste aversion conditioning. Bulletin of the Psychonomic Society, 20, 101-104.

Palmerino, C. C., RusiniaK, K. W., \& Garcia, J. (1980). Flavorillness aversions: The peculiar roles of odor and taste in memory for poison. Science, 208, 753-755.

Pavlov, I. P. (1927). Conditioned reflexes (G. V. Anrep, Trans.). Oxford: Oxford University Press.

Pearce, J. M. (1987). A model for stimulus generalization in Pavlovian conditioning. Psychological Review, 94, 61-73.

Rescorla, R. A., \& Wagner, A. R. (1972). A theory of Pavlovian conditioning: Variations in the effectiveness of reinforcement and nonreinforcement. In A. H. Black \& W. F. Prokasy (Eds.), Classical conditioning II: Current research and theory (pp. 64-99). New York: Appleton-Century-Crofts.

Rusiniak, K. W., Hankins, W. G., Garcia, J., \& Brett, L. P. (1979). Flavor-illness aversions: Potentiation of odor by taste in rats. $B e$ havioral \& Neural Biology, 25, 1-17.

Rusiniak, K. W., Palmerino, C. C., Rice, A. G., Forthman, D. L., \& Garcia, J. (1982). Flavor-illness aversions: Potentiation of odor by taste with toxin but not shock in rats. Journal of Comparative \& Physiological Psychology, 96, 527-539.

SClafani, A. (1987). Carbohydrate taste, appetite, and obesity: An overview. Neuroscience \& Biobehavioral Reviews, 11, 131-153.

Terrace, H. S. (1966). Stimulus control. In W. K. Honig (Ed.). Operant behavior: Areas of research and application (pp. 271-344). New York: Appleton-Century-Crofts. 